
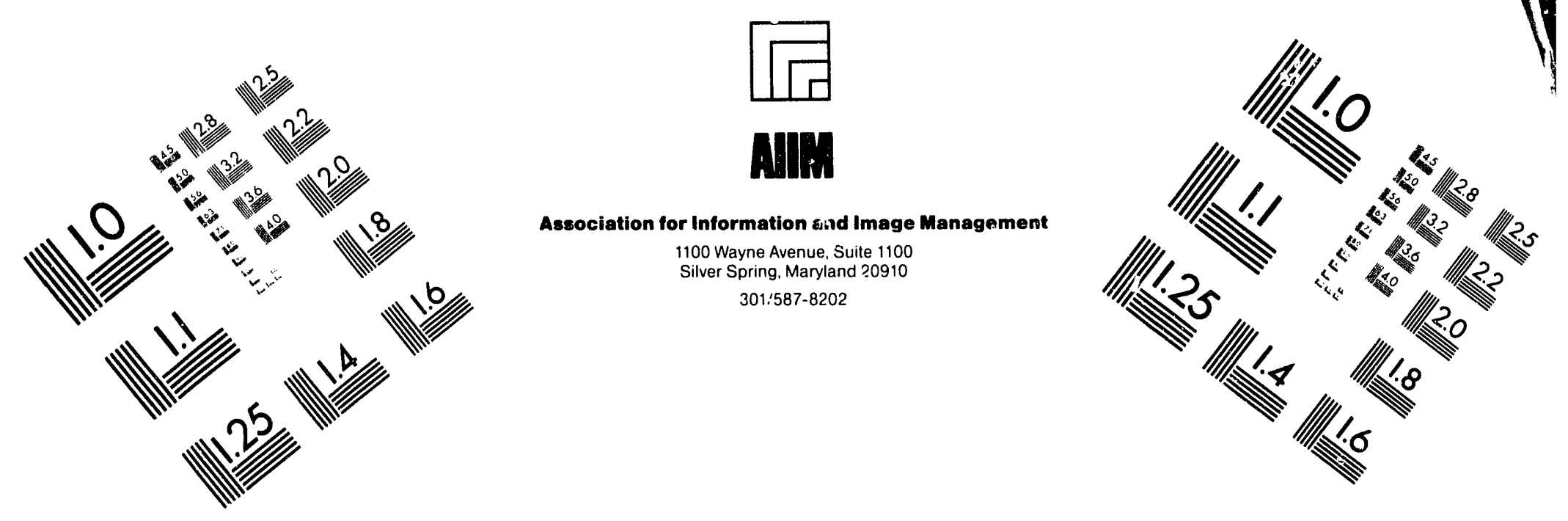

Centimeter

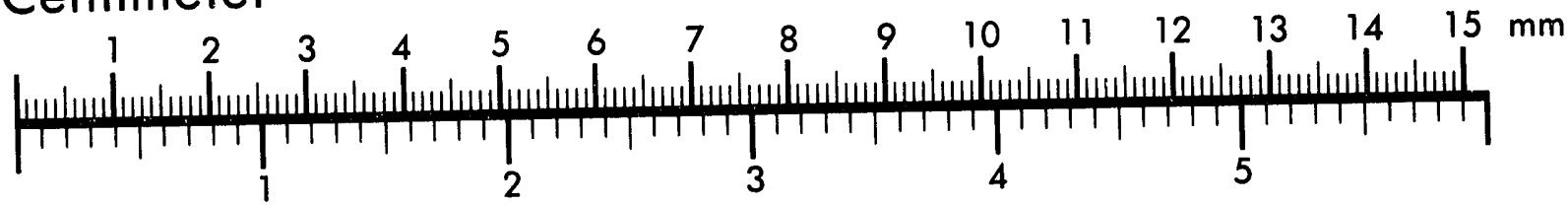

Inches
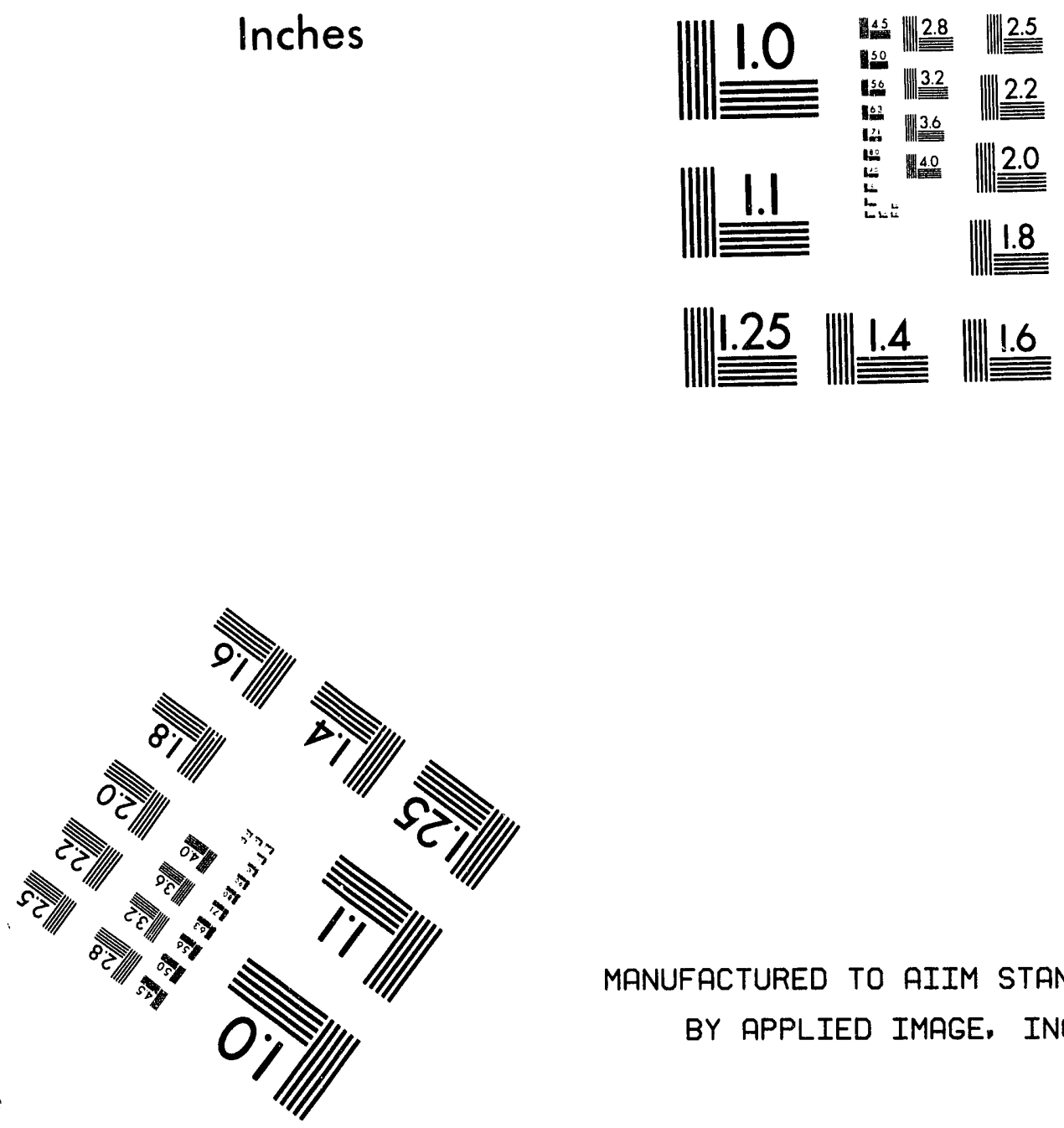

MANUFACTURED TO AIIM STANDARDS

BY APPLIED IMAGE, INC.

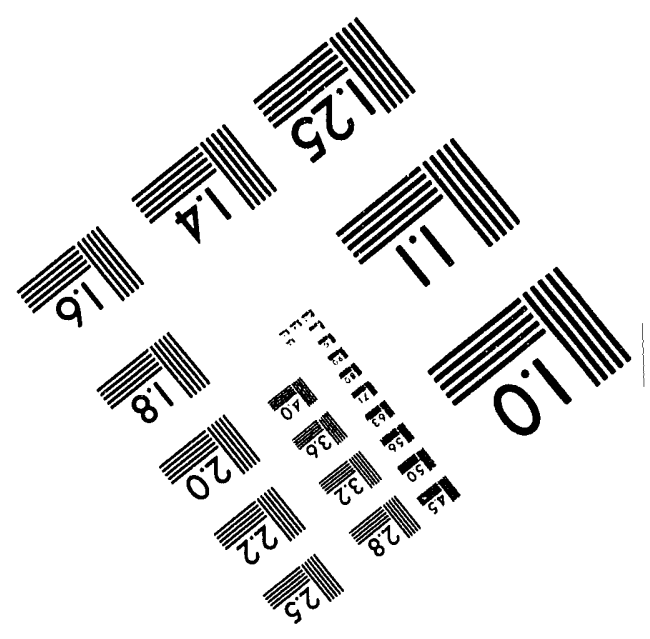



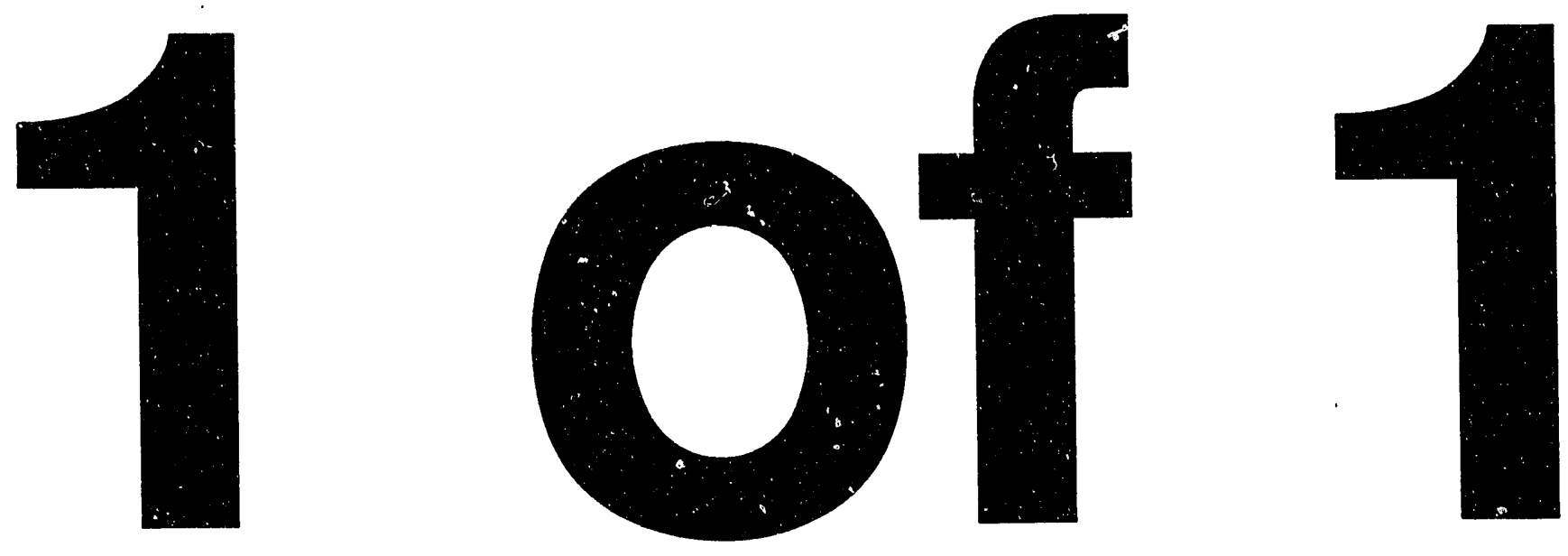


\title{
Optimization Method for Orbit Correction in Accelerators:
}

\author{
Eva Bozoki and Aharon Friedman \\ National Synchrotron Light Source, \\ Brookhaven National Laboratory, Upton, NY 11973
}

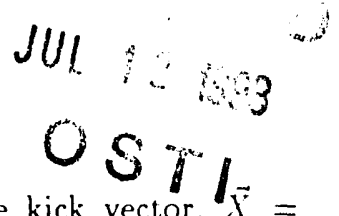

\section{Abstract}

We present a method to minimize the corrector strengths required to reduce the rms beam orbit. Any least square correction method will usually lead to undesirably strong corrector settings. The method, we are presenting, minimizes the total kick vector by finding the eigen solutions of the equation $\vec{X}=A \hat{\theta}$, where $\vec{X}$ is the orbit change vector $\hat{\theta}$ is the kick vector and $A$ is the response matrix. Since $A$ is not necessarily a symmetric or even square matrix we symetrize the matrix by using $A^{T} A$ instead. Eigen vectors with corresponding small eigen values generate negligible orbit changes. Hence, in the optimization process the kick vector is made orthogonal to the eigen vectors.

The physicai interpretation of the eigenvectors will be discussed. We will illustrate the application of the method to the NSISS X-ray and UV storage rings. From this illustration it will be evident, that the accuracy of this method allows the combination of the global orbit correction and local optimization of the orbit for beamlines and insertion devices.

\section{Introduction}

In circular machines, the beam orbit is usually very important to the output of the machine. A good example is synchrotron radiation facilities where the source point and direction of the photon beams depend on the beam position and angle. In addition the lifetime of the beam in the machine, the maximum current, and sometimes the ability of the machine to store a beam at all depends on an accurate beam orbit.

\section{The response matrix and its eigen states}

Usually in a circular machine, the beam position is monitored by a set of pickup electrodes (PUE), distributed around the machine and orbit correction is performed using a set of distributed dipoles (correctors). The relationship between a charge in the strength of a corrector and the corresponding change of the beam position at the PUEs, is expressed by the response matrix. In this section we define and analyze the response matrix.

\subsection{Definition of the response matrix}

It is well known that the orbit change due to a change in the corrector strengths (ortit kick) call be expressed as [1]:

$$
\vec{X}=A \vec{\Theta}
$$

- Work performed under the auspices of (he U.S. Dept. of Energy

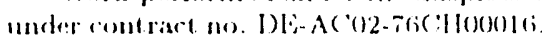

where $\vec{\Theta}=\left[\Theta_{j}\right], 1 \leq j \leq N_{c}$ is the kick vector, $\vec{X}=$ $\left[X_{i}\right], 1 \leq i \leq N_{m}$ is the orbit vector and $A=\left(A_{i, j}\right)$ is the response matrix. The element $A_{i, j}$ of the response matrix is the orbit change on the $i$-th orbit monitor due to a unit kick from the $\mathrm{j}$-th corrector. In this paper, unless otherwise specified, the beam position and the orbit kick are expressed in $\mathrm{mm}$ and $\mathrm{Kdigit}^{1}$, respectively.

\subsection{Eigen solutions of the response matrix}

In general, the number of correctors and monitors are different, consequently the response matrix $A$ is rectangular and Eq. (1) is over or under constrained. To solve Eq. (1), we left-multip!y it by $A^{T}$, the transpose of the response matrix $A$ :

$$
A^{T} \vec{X}=A^{T} A \vec{\Theta} .
$$

The solution of Eq. (2) is the least-square approximation of a solution of Eq. (1). The matrix $A^{T} A$ is symmetric and non negative. The eigen solutions of $\mathrm{Eq}$. (2) can be found by diagonalyzing the matrix $A^{T} A$, resulting in the eigen values $\left[\lambda_{j}\right]$ and the corresponding eigen vectors $\left[\hat{\theta}_{j}\right]$. An eigen vector $\hat{\theta}_{j}$ represents a set of corrector values. The orbit change corresponding to the $j$-th eigen vector is:

$$
\vec{x}_{j}=A \hat{\theta}_{j} .
$$

It can be shown that

$$
\left(\vec{x}_{j} \cdot \vec{x}_{j}\right)=\lambda_{j}
$$

The physical meaning of the eigen values becomes clear from Eq. (4). An eigen value $\lambda_{j}$ is a quantitative measure of the orbit response (of the machine) to the $j$-th eigen vector. A small eigen value $\lambda_{j}$ corresponds to a small $x_{j}$ orbit change $\left(\left\langle x_{j}{ }^{2}\right\rangle_{r m s}=\lambda_{j} / N_{m}\right)$. This property of the eigen solutions will be used later in the paper for reducing the corrector strengths without significantly changing the resulting orbit.

\section{The representation of a circular machine}

In order to give physical interpretation to the response matrix and its eigen solution one has to look at the dynamics of the beam orbit in a circular machine. In this section we lay out the dynamics that lead to the response matrix and show the physical meaning of its eigen solution in one casse.

\footnotetext{
'digits - Digitized voltage of the computer controlled corrector puwer supply.

DISTRIBUTION OF THIS DOCUMENT IS UNLIMITE
} 


\section{DISCLAIMER}

This report was prepared as an account of work sponsored by an agency of the United States Government. Neither the United States Government nor any agency thereof, nor any of their employees, makes any warranty, express or implied, or assumes any legal liability or responsibility for the accuracy, completeness, or usefulness of any information, apparatus, product, or process disclosed, or represents that its use would not infringe privately owned rights. Reference herein to any specific commercial product, process, or service by trade name, trademaik, manufacturer, or otherwise does not necessarily constitute or imply its endorsement, recommendation, or favoring by the United States Government or any agency thereof. The views and opinions of authors expressed herein do not necessarily state or reflect those of the United States Government or any agency thereof. 


\subsection{Courant-Snyder equation}

For a given set of discrete PUEs and correctors, the beam position at the $j$-th PlE is:

$$
\frac{x_{i}}{\sqrt{\beta_{i}}}=\frac{1}{2 \sin \pi \nu} \sum_{j=1}^{N_{\mathrm{c}}} \theta_{j} \sqrt{\beta_{j}} \cos \nu\left(\left|\phi_{i}^{\prime}-\phi_{j}\right|-\pi\right),
$$

where $\beta_{i}$ is the $\beta$ function value at the PUE in which $x_{i}$ is observed, $\phi_{i}^{\prime}$ is the phase location of that $\mathrm{PUE}, \theta_{j}$ is the angular kick introduced to the beam by the corrector located at phase $\phi_{j}, \beta_{j}$ is the $\beta$ function value at $\phi_{j}, \nu$ is the tune (number of betatron oscillations) of the machine, and $N_{c}$ is the total number of correctors.

It is easy to see from Eq. (5) that the elements of the response matrix $A$ are:

$$
A_{i j}=\frac{\sqrt{\beta_{i} \beta_{j}}}{2 \sin \pi \nu} \cos \nu\left(\left|\phi_{i}^{\prime}-\phi_{j}\right|-\pi\right) .
$$

Note, that in most practical cases the numerical value of the matrix element $A_{i j}$ cannot be theoretically evaluated to a satisfactory accuracy, since the values of $\beta_{i}$ and $\beta_{j}$ are not known. It was actually suggested [4] to use the measured value of the response matrix elements in order to estimate the values of the $\beta$ function.

\subsection{Equidistant correctors and monitors}

Consider the case, when there are equal number of PUEs and correctors $\left(N_{m}=N_{c}=N\right)$ and they are positioned at equal intervals around the ring. Furthermore, for the sake of simplicity, we assume that the $\beta$ function has the same value for any $\operatorname{PUE}\left(\beta_{i}=\beta_{M}\right)$, and that it has the same value for any corrector $\left(\beta_{j}=\beta_{C}\right)$. Clearly the response matrix in such a case is cyclic ${ }^{2}$ since the symmetry is such that the point $i=0$ can be chosen arbitrarily to be any PUE. It is indeed, easy to prove, by way of mathematical induction that Eq. (6) yields a cyclic matrix whose first line is

$$
A_{1 j}=\frac{\sqrt{\beta_{M} \beta_{C}}}{2 \sin \pi \nu} \cos \nu\left[\frac{2 \pi}{N}\left(j-\frac{1}{2}\right)-\pi\right] .
$$

The $i$-th element of the $j$-th eigen vector and the $j$-th eigen value of a cyclic matrix $A$ are:

$$
\begin{gathered}
\hat{E}_{j}(i)=\frac{1}{\sqrt{N}} \operatorname{cxp}\left(i \frac{2 \pi}{N} j \nu\right), \\
\lambda_{j}=\sum_{k=1}^{N} A_{k} \exp \left[i \frac{2 \pi}{N} j(k-1)\right] .
\end{gathered}
$$

Thus the eigen vecters of the response matrix are those expressed by Eq. (8), mammly they are the harmonics of

\footnotetext{
${ }^{2} A$ ryclic: matrix is a matrix where the limes ane arrangerl so that the first element of a line is the list plement of the previous line and the ather clemensts are coppers from the previous line.
}

the ring. Substituting Eq. (7) in Eq. (9) results in

$$
\begin{aligned}
\lambda_{j} & =\frac{1}{2} \exp \left[i \pi j\left(1-\frac{1}{N}\right)\right] \frac{\sin \pi(j+\nu)}{\sin \frac{\pi}{N}(j+\nu)} \\
& +\frac{1}{2} \exp \left[-i \pi j\left(1-\frac{1}{N}\right)\right] \frac{\sin \pi(j-\nu)}{\sin \frac{\pi}{N}(j-\nu)}
\end{aligned}
$$

As it is expected [1], the orbit response to the $j$-th harmonic $\left(\lambda_{j}\right)$ gets larger as $j$ gets closer to the tune $\nu$.

It can be shown [5], that for the most general case of non symmetric rings with non equidistant and non equal number of correctors and monitors, the eigenvectors represent the harmonics, the local bumps and the errors in the ring.

\section{Corrector strengths reduction}

As mentioned earlier, the property of the eigen solutions that a small eigen value corresponds to a small orbit change can be used for reducing the corrector strengths without significantly changing the resulting orbit.

Let $\vec{X}_{o}$ be the orbit to be corrected and $\vec{\Theta}$ its corresponding kick vector, calculated by any method (e.g. least square). The RMS of the residual orbit is:

$$
X_{r m s}^{2}=\frac{1}{N_{m}}\left|A \vec{\Theta}-\vec{X}_{o}\right|^{2} .
$$

As long as the $\lambda_{j}$ eigenvalue is small, the corresponding $\vec{x}_{j}$ orbit is small and one can reduce the $\vec{\Theta}$ kick vector by the $j$-th eigenvector without significantly modifying the orbit. The reduced kick vector is:

$$
\begin{gathered}
\vec{\Theta}_{r e d}=\vec{\theta}-\sum_{j=1}^{M}\left(\vec{\Theta} \cdot \vec{\theta}_{j}\right) \vec{\theta}_{j}, \\
\Delta \vec{X}_{r m s}^{2}=\vec{X}_{r m s}^{2}-\left(\vec{X}_{r e d}^{2}\right)_{r m s}<\epsilon .
\end{gathered}
$$

The $\vec{\theta}$ kick vector is 'equivalent' to the original $\vec{\Theta}$ vector to $c$ accuracy. It is important, that both, the norm of the vector and its largest component is reduced:

$$
|\vec{\theta}|^{2}<|\vec{\Theta}|^{2} \text { and } \theta_{\max }<\Theta_{\max } \text {. }
$$

This method was implemented in the NSLS resulting in reduction of up to $70 \%$ in the average corrector strength and up to $90 \%$ in the maximum corrector strength. In addition the accuracy of orbit correction was significantly improved since the elimination of the small eigenvalue states reduces the crror in orbit calculation.

\section{Correction by decomposition}

In the previous Section, we made use of the eigensolutions to reduce a given $\vec{\Theta}$ kick vector, obtained by any method of orbit correction. However, one can directly use an eigenvector decomposition based orhit correction method, thus avoiding the meed for reduction. This method will yield the 'minimum' kick vector for a desired accuracy of orbit. correction. 


\subsection{Global orbit correction}

Let $-\vec{X}_{o}$ be the orbit to be corrected and let us decompose it in terms of the $\vec{x}_{j}$ 'eigen' ${ }^{\prime 3}$ orbits:

$$
c_{j}=\vec{X}_{0} \cdot \vec{x}_{j}
$$

That is, each $\vec{x}_{j}$ vector is represented in $\vec{X}_{o}$ by the $c_{j}$ coefficient.

Let us next define an orbit vector, $\vec{X}_{\epsilon_{0}}$ as:

$$
\vec{X}_{\epsilon_{0}}=\sum_{j=1}^{N_{c}} c_{j} \cdot \vec{x}_{j}
$$

The norm of this vector is $\left|\vec{X}_{\epsilon_{o}}\right|^{2}=\sum_{j=1}^{N_{c}} c_{j}$ and $\left|\vec{X}_{\epsilon_{o}}\right|^{2} \leq$ $\left|\vec{X}_{o}\right|^{2}$. Actually, the uncorrectable part of the orbit is: ${ }^{4}$

$$
\Delta \vec{X}_{r m s}^{2}=\frac{1}{N_{m}}\left(\left|\vec{X}_{o}\right|^{2}-\left|\vec{X}_{\epsilon_{o}}\right|^{2}\right)=\epsilon_{o}^{2} .
$$

Substituting Eq. (3) into Eq. (16) we obtain

$$
\vec{X}_{\epsilon_{0}}=A \sum_{j=1}^{N_{c}} c_{j} \hat{\theta}_{j}
$$

that is, the kick vector which corrects the $\vec{X}_{o}$ orbit to to $\epsilon_{0}$ accuracy can be obtained from the eigenvector decomposition of this orbit as:

$$
\vec{\Theta}=\sum_{j:=1}^{N_{c}} c_{j} \hat{\theta}_{j} / \sqrt{\lambda_{j}}
$$

This method already assured that we are using only the minimum contribution from each eigenvector, thus the $\vec{\Theta}$ kick vector is 'minimized'. If, however, one can allow an $\epsilon>\epsilon_{o}$ tolerance in the orbit correction, then some eigenvectors (or part of it) with the smallest eigen values can be omitted, further reducing the $\vec{\Theta}$ kick. Actually, we can skip the eigenvectors until the corresponding decomposition coefficients satisfy:

$$
\sum_{j=1}^{J} c_{j}{ }^{2} \leq E \quad \text { where } E \equiv N_{m} \epsilon^{2} .
$$

In reality, there is no such $J$, for which the equality would be exactly satisfied. Generally, the sum for the first $J-1$ eigenvector is $\mathrm{E}^{\prime}<\mathrm{E}$ and for the $J$-th eigenvector the sum will be greater then $\mathrm{E}$ :

$$
\sum_{j=1}^{J-1} c_{j}^{2}+c_{J}^{2}=E^{\prime}+c_{J}^{2}
$$

\footnotetext{
${ }^{3}$ The $\vec{x}$, vectors are defined in by. (3), they represent the orbit change corresponding to the $\dot{\theta}$ eigenvecturs. They comprise in orthogonal but not a complete ortogantal set, and they are mot unit vectors.

4 'This part of the orbit cannot be corrected by any method with the given set of orbit correctors.
}

Therefore to achieve an $\epsilon$ accuracy in the orbit correction, we can omit the first $J-1$ eigenvectors and a part of the $J$-th. The remaining terms will be:

$c_{J}^{\prime 2}+\sum_{j=1}^{J+1} c_{j}^{2} \quad$, where $c_{J}^{\prime}=c_{J}-\Delta \quad$ and $\quad \Delta= \pm \sqrt{E-E^{\prime}}$

and the sign of $\Delta$ is the same as the sign of $c_{J}$.

The implementation of this method in the NSLS resulted in orbit correction to an accuracy of $X_{r m s}=15 \mu \mathrm{m}$ with very small changes in corrector strength (sometimes the average corrector strength was in the single digits).

\subsection{Including local bumps}

The tolerance on the global RMS orbit, even though very stingent, is usually larger then the tolerance on a few selected PUE's, usually at the ends of insertion devices. In case of the NSLS X-ray ring, for example, the requirement on the global RMS ortit is $100 \mu$, while before/after the insertion devices $20 \mu$. During operation, for each fill of the ring, first the global orbit was corrected using harmonic or least-square method then local bumps were implemented to position the orbit more accurately at the insertion devices.

With the decomposition method ve implemented the global and local orbit correction at the same time by assigning different weight factors at those PUE's in sensitive positions before/after the insertion devices.

\section{Other uses of eigen vectors}

One can use the Eigen vectors to measure the Response Matrix. Instead of the usual method of kicking with one corrector at a time and measuring the orbit response of the ring, the excitation of the Eigen vectors yield better results with higher accuracy [5].

We are also using the Eigen vector decomposition method in Digital Feedback in the storage rings (see [6]).

\section{REFERENCES}

[1] G. Guinard, CERN 77-10 ISRD, 6 June (1977).

[2] A. Jackson, SRS/NSS/75/103 (1975).

[3] E.D. Courant and H.S. Snyder, Annals of Phys., 3, No. 1, p. 1-48, (1958).

[4] E. Bozoki, Proc. of the Workshop on Modrling Based Accelerator Controls, p. 5, (1987).

E. Bozoki, NSLS Tech Notes \#291 and \#296, (1987).

[5] A. Friedman and E. Bozoki, In preparation.

[6] A. Friedman, E. Bozoki, O. Singh, J. Smith, In this Proccedings. 

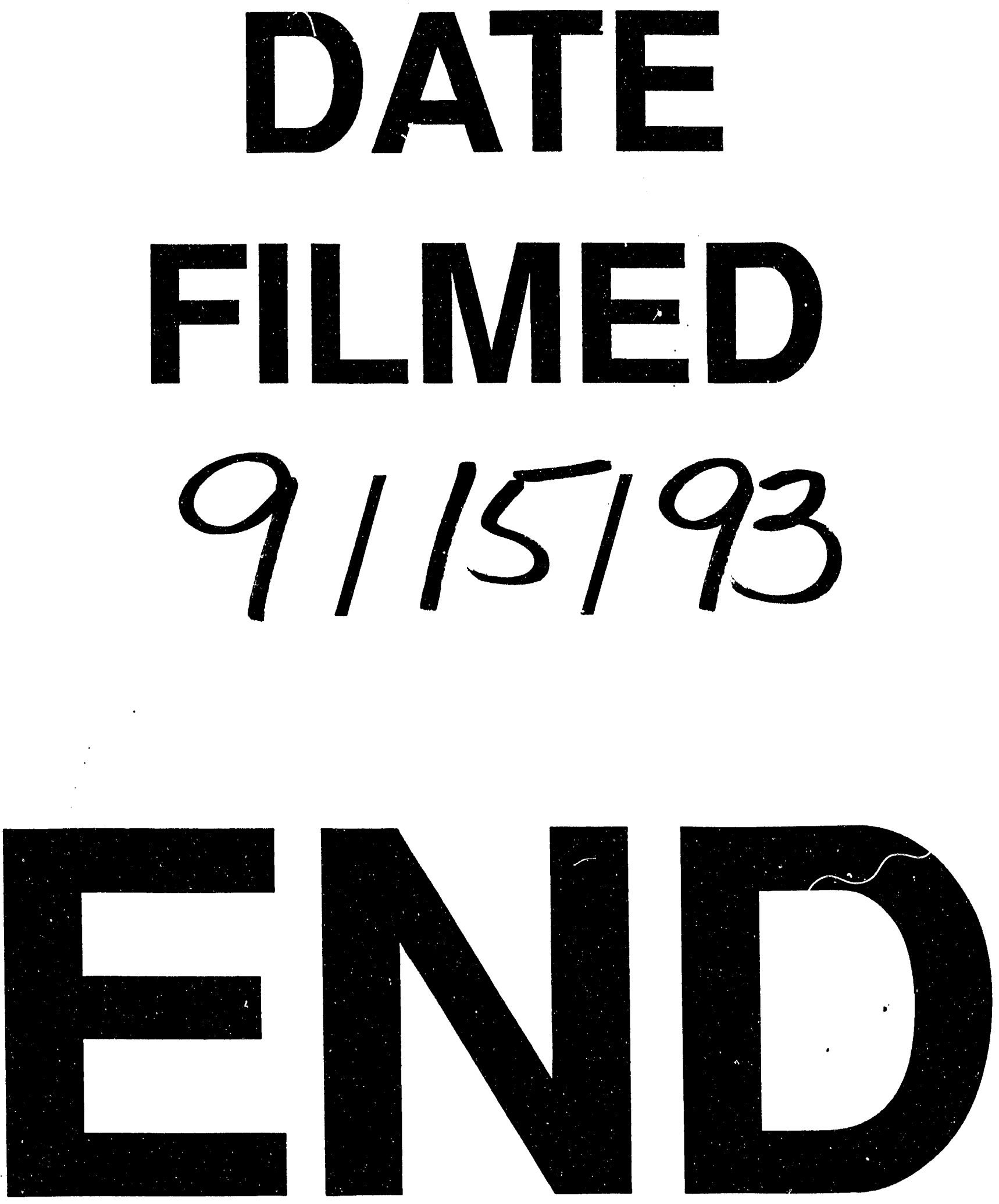


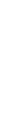

